\title{
Establishing a Framework to Explore the Servicer-Client Relationship in On-Orbit Servicing
}

Tiago Henrique Matos de Carvalho*, Jennifer Kingston

Cranfield University

College Road, Cranfield, Bedfordshire, United Kingdom MK430AL

* Corresponding Author

Email addresses: t.h.matosdecarvalho@cranfield.ac.uk (T. Matos de Carvalho), j.kingston@cranfield.ac.uk (J. Kingston)

\begin{abstract}
On-Orbit Servicing (OOS) is becoming more extensively discussed in research and commercial developments. The key point of servicing relies on the relation between two systems, the Servicer executing the tasks and the Client being serviced. With the increase of interest in OOS applications, the exploration of the Client-Servicer relationship in different scenarios becomes a necessary step in servicing implementation. Exploring such a relationship involves translating the information available about OOS into useful inputs for spacecraft design, a challenging task due to the variety of applications and possible interactions between both sides. Then, different modelling and simulation techniques can be used to model and to explore the interaction of such complex systems for different scenarios of servicing. This paper presents an agent-based framework to explore the relations of Servicer and Client for applications of On-Orbit Servicing. To do so, the case for a hypothetical satellite operator is explored, under a specific set of requirements and conditions for different servicing solutions. First, the Agent Based Modelling and Simulation (ABMS) is established based on a specific set of rules, definition metrics and operation characteristics relating Servicer and Client. Then, the basic assumptions of Client, Servicer and servicing operations are discussed and the initial simulation parameters are defined. The ABMS is used to simulate OOS for a fleet of geostationary communication satellites for the cases of Lifetime Extension, Refuel and Rescue and Recover. In the end, the ABMS outputs are used to explore the design aspects of the Servicer and Client satellites at systems level. The results highlight the advantages of having this type of framework for early assessment of OOS under different types of context for both Servicer and Client. The paper concludes with directions of how the framework can be used to explore more complex and realistic scenarios of OOS, and assess their potential benefits.
\end{abstract}

Keywords: On-Orbit Servicing, Servicer/Client Relation, Agent Based Model

\author{
Nomenclature \\ $\theta$ : Weibull scale parameter in years \\ $\beta$ : Weibull shape parameter \\ $t$ : time \\ $T_{\text {obs }}:$ Time to obsolescence of a system \\ $t_{\text {life }}$ : Initial design life of a satellite \\ TTBE: Time to break even \\ $\Delta V: \Delta V$ metric \\ $R(t):$ Reliability metric \\ NPV: Net Present Value metric \\ TTBE: Time to Break Even \\ $r$ : Discount rate
}

\section{Acronyms/Abbreviations}

ABMS - Agent Based Model and Simulation

COSEMS - Comprehensive Operational Support Evaluation Model for Space

DTA - Decision Tree Analysis

DES - Discrete Event Simulation

MEV - Mission Extension Vehicle

OOS - On-Orbit Servicing

RSGS - Robotic Servicing of Geosynchronous Satellites

RPO - Rendezvous and Proximity Operations

TRL - Technology Readiness Level

\section{Introduction}

On-Orbit Servicing (OOS) is one solution that has been explored throughout the history of space missions, aiming at the inspection, motion, maintenance, repair and assembly of a system in space. As space missions today are highly dependent on new solutions to better use the resources of a space system, especially from a commercial perspective, On-Orbit Servicing is clearly one option.

Examples of where this solution is needed start with space debris remediation (Active Debris Removal), going through extension of lifetime and maintenance of satellites to, finally, construction of larger infrastructures in orbit. 
More specifically in the robotic servicing area, considerable knowledge has been gained in the different areas/disciplines of servicing. As an example of this knowledge, Figure 1 shows a timeline adapted from Matos de Carvalho and Kingston [1] with developments of systems and spacecraft aimed for robotic OOS. Such timeline complements reviews previously presented by Flores-Abad et al. [2] and Gefke and Reed [3] in the field of space robotics for OOS. However, such solution is still not fully commercially operational despite being demonstrated a few times. The full implementation of servicing depends on the successful interaction of the Servicer, the system executing all the tasks, and the Client, the system receiving the service.

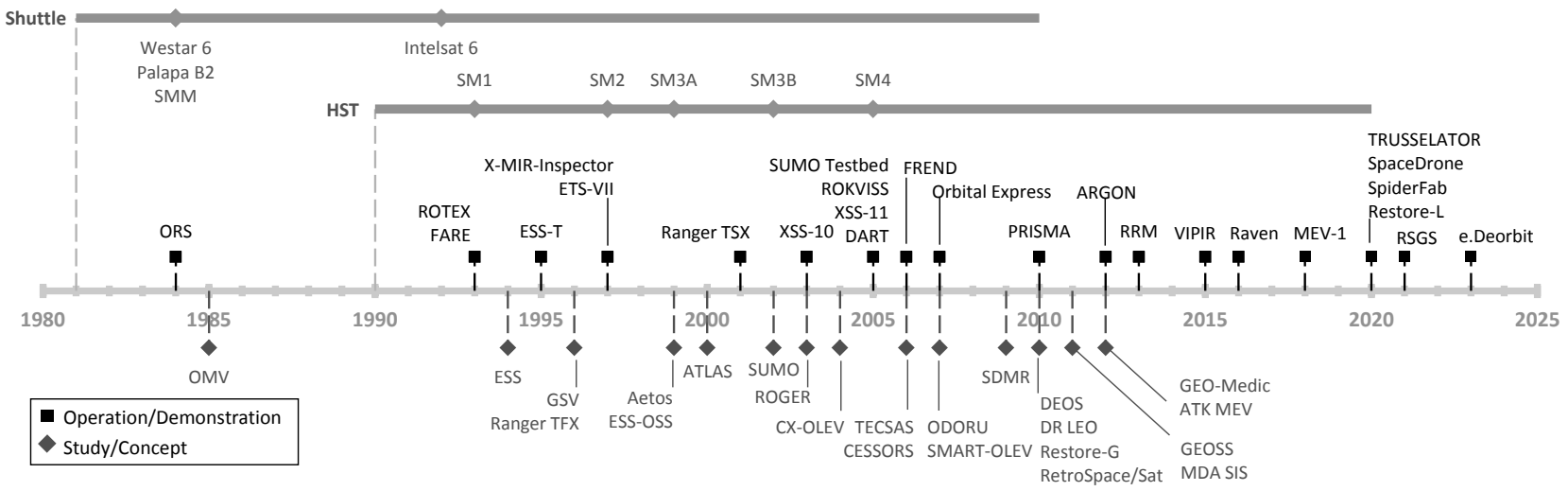

Fig. 1. On-Orbit Servicing - Developments Timeline (adapted from [1])

Today, OOS is presented with a sufficient maturity to be implemented effectively for basic applications such as inspection and motion tasks.

As the related research pushes the maturity of more advanced servicing applications towards an implementation stage (TRL >7) and demonstrates the benefits of servicing, companies start to openly express interest in OOS. To date, the following OOS missions are scheduled to be launched within the next five years:

Table 1. Commercial OOS missions

\begin{tabular}{lllll} 
Servicer & Client & Type & Launch & Ref \\
\hline MEV & Intelsat & Life. Ext. & 2018 & {$[4,5]$} \\
SpaceDrone & Undisclosed & Life. Ext. & 2020 & {$[6]$} \\
Restore-L & Landsat-7 & Refuel & 2020 & {$[7]$} \\
RSGS & SES & Refuel and Life. Ext. & 2021 & {$[8]$} \\
SpaceTug & Undisclosed & Refuel and Life. Ext & Undisclosed & {$[9]$}
\end{tabular}

Additionally, the steps towards commercial OOS are also complemented by developments to support more advanced servicing applications. Such developments can range from the concept definition of a next generation infrastructure to accommodate OOS [10,11], the potential market and impact of OOS [12] up to the proposition of a modular and universal interface such as iBOSS [13,14].

Within this context, this paper presents the relationship approach and the agent based framework to model and explore the Client and Servicer relation under a specific set of requirements and conditions for OOS. This provides assistance to a hypothetical satellite operator who may be considering if, when, and how to use OOS, and also to a hypothetical OOS provider, when considering the optimum services to offer.

As the current OOS programmes suggest, the interest of GEO operators is one of the main drivers now. Based on this, the example presented in this paper simulates the interaction of a given fleet of geostationary communication satellites and a fleet of Servicers. The OOS applications currently considered are those with most commercial appeal:

- Lifetime Extension: Assistance for station keeping or correction manoeuvres after depletion of a Client's propellant or when depletion is imminent. In this case the Servicer stays attached/docked with the Client for a given time providing the orbital manoeuvres/corrections necessary, extending the operation of the Client. This application can be planned in advance. As the upcoming OOS missions suggest [9,15-17], the Servicer will have the capability of moving between Clients to service them consecutively instead of staying in one single Client for the whole extension period. As such an approach would demand a more complex exploration of which Clients to service, the case presented here considers a Servicer dedicated to one single Client per time. Later in the paper this assumption is discussed in detail compared to the approach to be used by the missions in Table 1.

- Refuel: Renewal of the Client's fuel. Like Lifetime Extension, the Refuel application allows the Client satellite to operate for a longer period. However, in this case the Servicer moves to the Client`s orbital slot and transfers a given amount/mass of fuel. After the fuel transfer the Servicer is available to refuel a different Client satellite. This application can also be planned in advance.

- Rescue and Recover: Orbit insertion, correction or positioning and mechanisms deployment. This application is a response to a failure and cannot be planned in advance. 


\subsection{Relationship Approach}

Within the research literature related to OOS, the majority was dedicated to the design of Servicer spacecraft $[2,18-$ 20], a small part to the design of Client spacecraft [21,22] but the relationship between them has only rarely been approached. The main reason is that translating the information available about OOS into useful inputs for spacecraft design could be challenging due to the variety of applications and possible interactions between both sides. To overcome this, the system-level view of this relation is necessary as a first step. The main idea of the Relationship Approach is to interact Servicer and Client, through their systems and functions, to better understand the capabilities of OOS applications. The interaction of Client and Servicer is proposed to identify emergent properties of this relation (for example operational constraints and systems design and operational requirements) and how such properties affect the design of both sides. Such properties can then be used for the readiness assessment of both Servicer main tasks/functions and Client systems and sub-systems, which enable the exploration of roadmaps for OOS, considering both sides. Additionally, this interaction feeds into the exploration of serviceable satellite designs for the Client side to be explored at early stages of a project.

The relationship can be represented using different modelling techniques. From the perspective of systems simulation Discrete Event Simulation (DES [23]) and Agent Based Modelling and Simulation (ABMS [23,24]) are techniques commonly used. The first is suggested to model a system operating through a chain of specific events from its entities; the modelling of a queue (system) in a bank, for example. The second is used to represent a more complex system and the interaction of the agents composing such system [25]; the modelling of a traffic grid system in a city, for example. Depending on the level of details and desired characteristics for the modelling, both methodologies would allow the characterization of an OOS environment. Agent Based Modelling and Simulation is used in this work in order to consider each agent (Client and Servicer satellites) as a single entity, and most important, because of the interaction of different agents of the system. Further considerations of each modelling methodology for systems simulation are discussed by Siebers et al. [23] and are beyond the scope of this paper.

In the 1970's, the Comprehensive Operational Support Evaluation Model for Space (COSEMS) [26] was developed by the USAF to explore different components for a responsive launch and operation infrastructure. This Discrete Event Simulation was not directly intended for OOS and was used to simulate an infrastructure in space (the system) encompassing, among other features, basic representations for refuelling and replacement of satellites. However, specific details of the entities (satellites and launchers) and how they interact are left aside. More recently, Richards [27] presented a simplified ABMS to explore the orbital transfers for OOS, focused in cases of failure. The model brings useful insights on points related to availability of Client satellites for cases of failure. However, the model is still focused on cases in which servicing is used as a response to a failure only. In addition, it does not account for specific relationship parameters for Servicer and Client such as Servicer capabilities and limitations, Client compatibility, degradation and system characteristics. From a customer-centric perspective, Lamassoure and Saleh et al. [28,29] present a framework focused on Decision Tree Analysis (DTA) to understand the value of flexibility and costeffectiveness of specific types of servicing, exploring in detail the uncertainty aspects faced by the Client regarding OOS. Yet, no specific relation of Client and Servicer is explored within that framework.

The framework proposed here (Figure 2) encompasses other factors that are critical to evaluate OOS in the current state. In addition to orbital manoeuvres, the space logistics, evaluation metrics and the knowledge of current OOS programmes provide the characterization of the "agents" of the model (Servicer and Client) as well as their "states", "relations" and the "environment" where they are operating. In this way, different servicing applications and scenarios can be simulated and analysed in early phases of mission design of both Servicer and Client. The outputs can be used to:

- Analyse satellite servicing plans and operation scheduling to understand how the launch time and the expected servicing will affect the fleet operation;

- Analyse the system overall degradation to understand which type of servicing fits better for the satellites of a fleet;

- Simulate eventual failures and analyse the overall fleet response with and without servicing;

- Perform initial trade-offs and design of required systems to accommodate different types of servicing;

- Concurrent elaboration and refinement of Client requirements and Servicer capabilities;

- Analyse the cost-benefit of servicing compared to a "classical" fleet management approach.

\section{Materials and Methods}

Three main steps are necessary for the definition of the framework and the simulation for the desired case. First, the mathematical part of the framework is modelled using the ABMS. The general rules and characteristics of Client and Servicer interaction are defined. Additionally, the main metrics used to describe the behaviour/degradation of the system with time are presented.

Then, the user needs and requirements are identified. This is made using hypothetical satellite operator based on the current largest operators of geostationary communication satellites. Using the SpaceTrak satellite database [30], their fleets are used as reference to define a fleet of 30 different satellites with different compatibility with OOS.

Finally, a set of initial characteristics are defined for the Servicer fleet and simulation conditions. The Servicer fleet is based on the systems currently in development, presented earlier.

The following sections describe each of the steps in detail. 


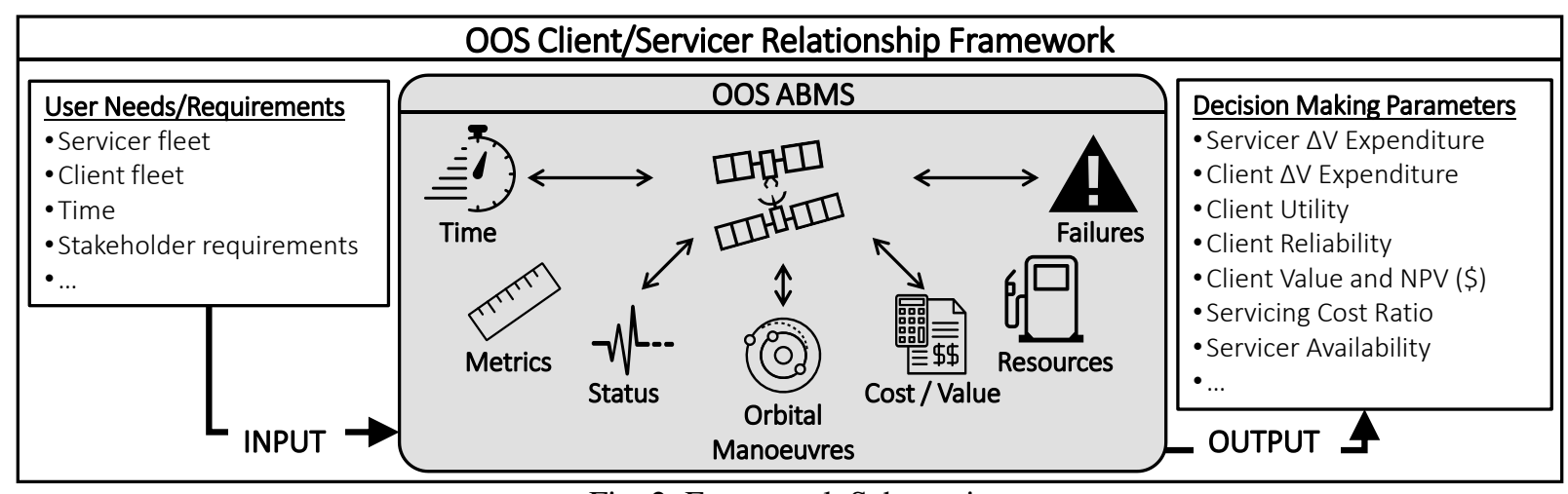

Fig. 2. Framework Schematic

\subsection{Agent Based Model}

The two main types of agents considered in this model are Client and Servicer. The general framework illustrated in Figure 2 works as follows:

1. The simulation runs for a given time $t$, considering a fleet of $n_{c}$ Client satellites and $n_{s}$ Servicer satellites. Based on the fleet definition from the Client satellite operator, Servicer satellites are spread in different orbital slots in the geostationary ring and they are each assigned one or more specific type of servicing capability.

2. Clients and Servicers start their operations at different times during the simulation. For Clients, this is defined by the operator's requirements. For Servicers this is defined by the servicing requests.

3. Client and Servicer satellites have a series of evaluation metrics to describe their states through the operational life. These metrics degrade with time; this will be described later.

4. Once one of the metrics reaches a minimum/limit value for one of the Client satellites, a servicing mission is assigned to the respective Client satellite, depending on the state and availability of the satellites of the Servicer fleet.

5. When a servicing operation is executed on one of the Client satellites, the changes in the system will affect the behaviour of the different metrics of the system. For the cases presented in this paper, servicing will affect the amount of fuel and the obsolescence time $\left(T_{o b s}\right)$. This is then reflected in the metrics dependent on these two parameters, including the financial aspects of each satellite.

6. Each servicing consumes resources from the Servicer and considers the time for the orbital manoeuvre, rendezvous and proximity operations (RPO), and a standard time for the servicing operation. Each Servicer remains in the vicinity of the last orbital slot until it is assigned to a new servicing.

7. The orbital manoeuvres are based on phasing manoeuvres for circular orbits [31,32]. Inclination changes are also accounted for in the manoeuvre calculations.

8. Client failures are simulated based on a failure rate per year (user input, currently based on real failure rates from SpaceTrak) and a stochastic generator to verify if a Client failed or not at a given time of the simulation.

9. If a Client requests any type of servicing but no Servicer can respond, the degradation will continue until either the system is serviced or until any of the metrics reach a critical level. In the second case the Client satellite is then considered dead.

10. If the Servicer runs out of fuel and is not able to be refuelled or if reaches the end of the design life it will be considered dead.

The following metrics and characteristics are described in detail:

- Reliability: This metric describes the probability of a system to survive after a given operational time [33]. It is applied to the satellites of the Client and Servicer fleet considering the Weibull scale $(\theta)$ and shape $(\beta)$ parameters from a population of GEO satellites from the year 2000 to 2017 (Appendix A). It degrades from the total reliability of the system (1) down to a residual reliability. By the end of life of a satellite operating for 15 years, the residual reliability can range from 0.8 to 0.9 [33].

$$
R(t)=e^{-\left(\frac{t}{\theta}\right)^{\beta}}
$$

- $\underline{V}$ : This metric describes the amount of $\Delta V$, in $[\mathrm{km} / \mathrm{s}]$, available for the satellites of the Client and Servicer fleet. It reduces from the total initial $\Delta V$ amount (on-station) down to 0 when the satellite is considered dead. A linear degradation is considered based on standard station keeping manoeuvres [34]. This metric (Equation 2) is affected by the satellite lifetime $\left(t_{\text {life }}\right)$, propulsion system characteristics and manoeuvres such as orbit-raising/apogee kick $\left(\Delta V_{\text {apogee }}\right)$, station-keeping $\left(\Delta V_{s k}\right)$ and phasing for Servicers. Auxiliary variables such as propellant mass $\left(m_{p}\right)$ for orbit-raising/apogee kick, operation (on-station) and for refuelling are calculated using the basic rocket equation (Equation 3), considering the respective $\Delta V$ needed, satellite total mass $\left(m_{0}\right)$ and dry mass $\left(m_{f}\right)$, in kilograms, and specific impulse $(I S P)$ in seconds.

$$
\begin{gathered}
\Delta V_{\text {total }}=\Delta V_{\text {apogee }}+\Delta V_{\text {life }}=\Delta V_{\text {apogee }}+\left(t_{\text {life }} \Delta V_{\text {sk }}\right) \\
\Delta V=g \operatorname{ISP} \ln \left(\frac{m_{0}}{m_{f}}\right)
\end{gathered}
$$


It is important to note that the use of low-thrust electric propulsion demands complex methods for estimation of $\Delta V$ necessary for orbit raising and station keeping. From the results presented by Dankanich and Woodcock [35], a $\Delta V$ ranging from $2.2 \mathrm{~km} / \mathrm{s}$ to $2.9 \mathrm{~km} / \mathrm{s}$ is estimated for electric orbit raising depending on the conditions considered. Losa [36] and Topputo and Bernelli-Zazzera [37] present different estimation methods using low-thrust for station keeping with results ranging from $69 \mathrm{~m} / \mathrm{s}$ to $180 \mathrm{~m} / \mathrm{s}$ per year. When using chemical propulsion, the rule of thumb for LEO-GEO orbit insertion and yearly station-keeping $\Delta V$ is around $1.5 \mathrm{~km} / \mathrm{s}$ and $50 \mathrm{~m} / \mathrm{s}$ per year respectively [34]. In both cases, the ratio between electric and chemical propulsion can be up to a factor of 2 . This factor is considered in the framework only for electric orbit raising due to the conditions of this phase of the mission, involving parameters such as thruster acceleration, atmospheric drag, solar pressure and manoeuvre duration. For stationkeeping it is assumed an estimation following the standard yearly $\Delta V$ requirements. Nevertheless, the $\Delta V$ capacity of Client or Servicer can be defined directly by the user if necessary and such information is known for a specific case.

- $\quad$ Net Present Value (NPV): This metric describes the amount of money generated by each Client satellite considering the Time Value of Money, stakeholder parameters for cash flow (operation costs and income) and system depreciation with time (obsolescence time* $T_{o b s}$ [38]). The net cash flow $C_{t}$ (Equation 4) is calculated using the expected cash income $\left(C_{\text {income }}\right)$ and operations cost $\left(C_{\text {opcost }}\right)$. Following the same process demonstrated by Graham et al. [39], the expected cash income $\left(C_{\text {income }}\right)$ is estimated via an iterative process using three stakeholder parameters: Cost as New $\left(C_{0}\right)$, Time to Break-Even** $(T T B E)$ and Discount Rate** $(r)$. The three parameters account for the capacity of a system to generate income, represented by the Equation 5, and can be adjusted to reflect a desired Client's operation scenario. Such values are treated as input variables provided by the user, otherwise default values are used.

$$
\begin{aligned}
& C_{t}=C_{\text {income }}-C_{\text {opcost }} \\
& N P V=\sum_{t=1}^{T} \frac{C_{t}}{(1+r)^{t}}-C_{0}
\end{aligned}
$$

* Although different methods to estimate the usefulness of a system and its obsolescence over time are available, herein the obsolescence is considered as a simple linear slope. At the beginning of life, the system is considered to have a maximum usefulness to the customer, which decreases based on a time to obsolescence defined by the user. This aspect is discussed in the last section.

**Time to Break-Even is used here as the time a satellite operator defines for a satellite to pay for itself from the revenues generated, and Discount Rate is the discount applied to the income with time used for a decision on the investment.

Table 2 presents the summary of all the primary variables required to explore a scenario using the framework. The variables are either used directly or/and compose the metrics described previously.

\begin{tabular}{|c|c|c|c|}
\hline Variable & Description & Remarks & Applicable \\
\hline$t_{\text {sim }}$ & Simulation time in years & - & - \\
\hline$n_{c}$ & Number of Clients & - & Client \\
\hline$n_{s}$ & Number of Servicers & - & Servicer \\
\hline$t_{\text {life }}$ & Design life in years & - & Client and Servicer \\
\hline$t_{\text {start }}$ & Operation start time in years & - & Client and Servicer \\
\hline$T_{o b s}$ & Obsolescence time in years & User defined (based on $t_{\text {life }}$ ) & Client \\
\hline$m_{\text {total }}$ & Total mass in $\mathrm{kg}$ & - & Client and Servicer \\
\hline prop $_{\text {life }}$ & Propulsion type for operation & $\mathrm{MP}, \mathrm{BP}$ or EP & Client and Servicer \\
\hline prop apogee & Propulsion type for orbit insertion & MP, BP, EP or none & Client and Servicer \\
\hline$\beta$ & Weibull scale parameter in years & Appendix A and [33] & Client and Servicer \\
\hline$\theta$ & Weibull shape parameter & Appendix A and [33] & Client and Servicer \\
\hline$\omega$ & Orbital slot (GEO) in degrees & - & Client and Servicer \\
\hline$i$ & Orbital inclination in degrees & - & Client and Servicer \\
\hline$a$ & Semi-major axis in $\mathrm{km}$ & - & Client and Servicer \\
\hline Orbit $_{\text {start }}$ & Starting orbit & GEO or GTO & Client and Servicer \\
\hline Cost & Cost as new in $\mathrm{M} \$$ & Includes launch cost & Client and Servicer \\
\hline Cost $_{\text {operation }}$ & Cost of operations per year in $\mathrm{M} \$$ & Default $=1 \% \operatorname{Cost}[34]$ & Client and Servicer \\
\hline Cost residual & Residual cost & As fraction of Cost & Client \\
\hline$T T B E$ & Time to Break-Even in years & - & Client \\
\hline$r$ & Discount rate & - & Client \\
\hline Compat $_{\text {serv }}$ & Desired type of servicing & Described in section 1 & Client \\
\hline$t_{\text {ext }}$ & Desired extension time & For Life. Ext and Refuel & Client \\
\hline$t_{\text {serv }}$ & Expected time to be serviced & As fraction of $t_{\text {life }}$ & Client \\
\hline Cost $_{\text {serv }}$ & Cost per resources $(\mathrm{M} \$ / \mathrm{kg})$ & Based on fuel spent/refuelled & Client and Servicer \\
\hline$m_{\text {prop-sell }}$ & Mass of sellable propellant in $\mathrm{kg}$ & For MP, BP or EP & Servicer \\
\hline \multirow{2}{*}{ rate $_{\text {failure }}$} & Maximum failures per year & For Rescue and Recover & Client \\
\hline & $\mathrm{BP}=\mathrm{Bi}$-propellant $\quad \mathrm{MP}=\mathrm{Mono}$ & ropellant $\quad \mathrm{EP}=$ Electric prop & sion \\
\hline
\end{tabular}

Table 2 - Primary variables used in the framework 
The variables related to the presented metrics are either provided by the user/stakeholder or assigned an assumed default value from the framework. This characteristic is discussed in the final section of the paper. Additionally, other characteristics of the model are defined as follows and have a direct effect over the metrics and agents' states.

- Compatibility: Client satellites are defined with compatibility to a given application of On-Orbit Servicing presented in Section 1. For cases of refuelling, only Servicers with a compatible refuelling system (propellant type) can respond to a servicing call.

- Self-maintenance: Servicer satellites designed for Refuel applications are considered serviceable for the current cases. This allows these satellites to be refuelled once they are moved to a specific orbital location considered for a "fuel depot" infrastructure. No other assumptions are made at this stage regarding the depot design, commissioning and operation from the perspective of the "Depot Operator".

- Charges: The Clients are charged by the Servicers according to the type of servicing application. During the simulation the model does not decide on a best charge for either Client or Servicer. The point of view adopted when using the model will define this characteristic; this is discussed later in the paper. Herein, the example assumes a Servicer operator aiming to pay for their systems by the end of the design life and a Client operator reaching better operational conditions when compared to the operation without any servicing. It should be noted that the definition of "better operational conditions" comes from the subjective view of each individual stakeholder.

The summary of the main characteristics of the ABMS and the rules is presented in Table 3.

Table 3. Summary of the OOS-ABMS

Servicer

Client

\begin{tabular}{lll}
\hline Agents & \multicolumn{1}{c}{ Fleet of $n_{s}$ size } & Fleet of $n_{c}$ size \\
Environment & GEO orbit for $t$ time in years & GEO orbit for $t$ time in years \\
States & Available & Operational \\
& Servicing & Non-operational \\
& Dead & Dead \\
Rules & Orbital manoeuvres & Reliability \\
& Servicing trigger & $\Delta V$ \\
& Servicing functions & Propellant Mass \\
& Available resources & Net Present Value
\end{tabular}

The whole framework is modelled using Excel spreadsheets running Visual Basic macros. Due to length limitations the code is not included in this paper. The flowchart of the main modules and processes of the framework is illustrated in Figure 3.

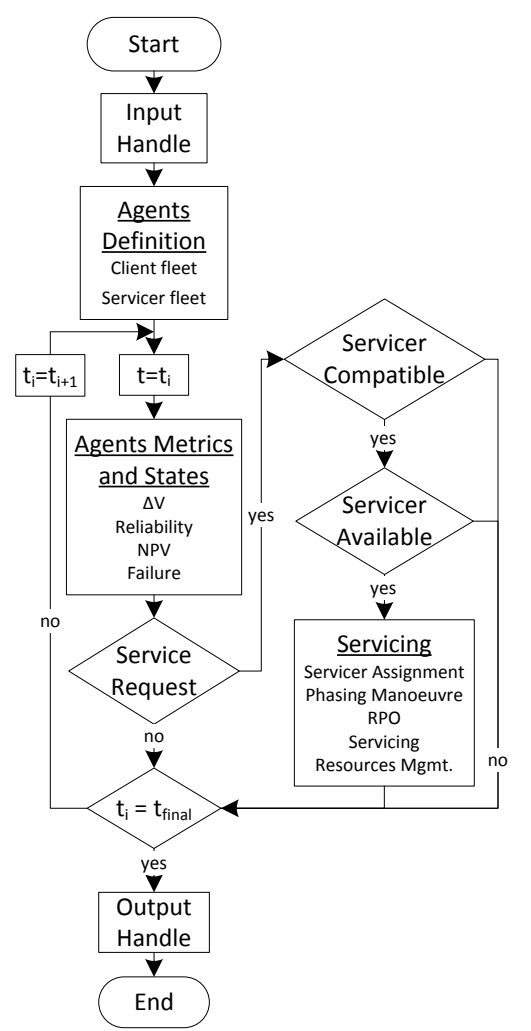

Fig.3 - OOS ABMS - Simulation Flowchart 


\subsection{Satellite Operator and Fleet Requirements}

A hypothetical case is considered here, to exemplify the use of the framework. The case considers an operator with a given fleet of geostationary communication satellites.

The three largest operators of GEO communication satellites (SES, Intelsat and Eutelsat) are used as a reference to define the fleet for this case. Characteristics such as orbital position, mass, satellites launched per year, and design life are extracted from the SpaceTrak database [30]. The sample has 124 satellites from all the three operators. The filters and options to collect the sample of satellites are defined in Table 4.

Table 4. SpaceTrak filters and options

\begin{tabular}{ll} 
Filter & Options \\
\hline Operator & SES S.A., Intelsat, Eutelsat S.A. \\
Launch Date & From 01/01/2000 to 31/12/2017 \\
Event Type & Launch - Successful \\
Sector & Commercial \\
Orbit Category & GEO \\
Spacecraft Status & Active
\end{tabular}

A total of 30 satellites are selected from the sample to compose the hypothetical operator fleet, representing a medium size fleet. Table 5 summarizes the satellites' characteristics for the Client fleet.

\begin{tabular}{|c|c|c|c|c|c|c|c|c|}
\hline \multirow[b]{2}{*}{ ID } & \multicolumn{8}{|c|}{ Table 5. Client fleet parameters } \\
\hline & $\begin{array}{c}\text { Total } \\
\text { Mass } \\
{[\mathrm{kg}]}\end{array}$ & $\begin{array}{c}\text { Mass On } \\
\text { Station } \\
{[\mathrm{kg}]}\end{array}$ & $\begin{array}{c}\text { Op. } \\
\text { start } \\
\text { [year] }\end{array}$ & $\begin{array}{c}t_{\text {life }} \\
\text { [years] }\end{array}$ & $\begin{array}{c}\text { Cost } \\
{[\mathrm{FY} 2018} \\
\mathrm{M} \$]\end{array}$ & $\begin{array}{l}\Delta V \text { On } \\
\text { Station } \\
{[\mathrm{km} / \mathrm{s}]}\end{array}$ & $\begin{array}{c}\text { Prop. } \\
\text { Orb. } \\
\text { Insert. }\end{array}$ & $\begin{array}{c}\text { Prop. } \\
\text { Station } \\
\text { Keep. }\end{array}$ \\
\hline 1 & 2784.0 & 1751.3 & 0.0 & 15 & 195.12 & 0.818 & $\mathrm{BP}$ & MP \\
\hline 2 & 4021.0 & 2529.5 & 8.1 & 15 & 255.57 & 0.818 & $\mathrm{BP}$ & MP \\
\hline 3 & 2649.0 & 1666.4 & 0.4 & 15 & 205.28 & 0.818 & $\mathrm{BP}$ & MP \\
\hline 4 & 2473.0 & 1555.7 & 11.9 & 16 & 180.29 & 0.872 & $\mathrm{BP}$ & MP \\
\hline 5 & 3903.0 & 2455.3 & 3.2 & 15 & 249.58 & 0.818 & $\mathrm{BP}$ & MP \\
\hline 6 & 1720.0 & 1082.0 & 2.1 & 12 & 152.12 & 0.654 & $\mathrm{BP}$ & EP \\
\hline 7 & 3901.0 & 2454.0 & 4.1 & 15 & 249.48 & 0.818 & $\mathrm{BP}$ & MP \\
\hline 8 & 1983.0 & 1247.4 & 4.0 & 15 & 158.24 & 0.818 & $\mathrm{BP}$ & MP \\
\hline 9 & 2015.0 & 1267.6 & 4.3 & 15 & 159.69 & 0.818 & $\mathrm{BP}$ & MP \\
\hline 10 & 4100.0 & 2579.2 & 6.7 & 15 & 273.49 & 0.818 & $\mathrm{BP}$ & EP \\
\hline 11 & 4332.0 & 2725.1 & 9.6 & 15 & 271.65 & 0.818 & BP & MP \\
\hline 12 & 3643.0 & 1894.8 & 4.8 & 15 & 241.02 & 0.818 & MP & EP \\
\hline 13 & 4385.0 & 2758.5 & 11.2 & 15 & 274.42 & 0.818 & $\mathrm{BP}$ & MP \\
\hline 14 & 2845.0 & 1789.7 & 1.0 & 15 & 197.99 & 0.818 & $\mathrm{BP}$ & MP \\
\hline 15 & 5922.0 & 3725.4 & 12.6 & 15 & 380.81 & 0.818 & $\mathrm{BP}$ & EP \\
\hline 16 & 4144.0 & 2606.9 & 0.0 & 15 & 275.9 & 0.818 & $\mathrm{BP}$ & MP \\
\hline 17 & 2205.0 & 1892.3 & 18.5 & 15 & 158.13 & 0.818 & $\mathrm{EP}$ & $\mathrm{EP}$ \\
\hline 18 & 2221.5 & 1906.4 & 19.8 & 15 & 158.13 & 0.818 & EP & $\mathrm{EP}$ \\
\hline 19 & 2500.0 & 1572.7 & 3.6 & 10 & 183.79 & 0.545 & $\mathrm{BP}$ & MP \\
\hline 20 & 5053.0 & 3178.7 & 9.3 & 16 & 327.76 & 0.872 & $\mathrm{BP}$ & EP \\
\hline 21 & 3551.0 & 3047.3 & 20.7 & 15 & 271.63 & 0.818 & $\mathrm{EP}$ & $\mathrm{EP}$ \\
\hline 22 & 4484.0 & 2820.8 & 3.3 & 15 & 294.77 & 0.818 & $\mathrm{BP}$ & $\mathrm{EP}$ \\
\hline 23 & 1760.0 & 1107.2 & 6.6 & 15 & 148.19 & 0.818 & $\mathrm{BP}$ & MP \\
\hline 24 & 4060.0 & 2554.0 & 7.1 & 15 & 271.31 & 0.818 & $\mathrm{BP}$ & EP \\
\hline 25 & 2087.0 & 1312.9 & 8.9 & 15 & 162.96 & 0.818 & $\mathrm{BP}$ & MP \\
\hline 26 & 2033.0 & 1278.9 & 9.1 & 15 & 261.63 & 0.818 & $\mathrm{BP}$ & MP \\
\hline 27 & 5493.0 & 3455.5 & 8.8 & 15 & 353.98 & 0.818 & $\mathrm{BP}$ & EP \\
\hline 28 & 4850.0 & 3051.0 & 5.8 & 15 & 315.66 & 0.818 & $\mathrm{BP}$ & EP \\
\hline 29 & 3300.0 & 2075.9 & 19.0 & 16 & 224.25 & 0.872 & $\mathrm{BP}$ & $\mathrm{EP}$ \\
\hline 30 & 2350.0 & 1478.3 & 11.3 & 15 & 174.97 & 0.818 & $\mathrm{BP}$ & MP \\
\hline & $\mathrm{BP}=\mathrm{Bi}-\mathrm{p}$ & pellant & MP & Mono-p & opellant & $\mathrm{EP}=\mathrm{Elec}$ & tric pro & llsion \\
\hline
\end{tabular}

The $\Delta V$ is estimated using total mass, design life and propulsive characteristics. Assuming a GTO to GEO transfer, a $\Delta V$ ranging from $1.5 \mathrm{~km} / \mathrm{s}$ [34] to $3 \mathrm{~km} / \mathrm{s}$ [35], depending on the propulsion type (chemical or electric), is considered 
and subtracted from the total $\Delta V$ calculated. The remaining $\Delta V$ is considered available for station keeping during the designed life.

Satellites' costs as new (including launch) are extracted from the SpaceTrak database when available, or calculated using cost estimation relationships (QuickCost and USCM8 [34]). Actual values can also be directly entered by a user if they are known.

The beginning of operation time is defined by checking the time the satellite was launched. All the satellites from the sample are sorted and the time is counted from the first Client satellite launched. Table 5 presents the main parameters for the satellites selected to compose the fleet for the case studied here.

Orbital slots for Client satellites considered are illustrated in Figure 4. The inclination is also included in the simulation despite being extremely small (not higher than 1 degree). However, the values are not presented here due to the minor relevance to this specific case.

The compatibility for each Client satellite is defined as:

- Lifetime Extension: Clients 6, 10, 12, 15, 18, 20, 21, 22, 24, 27, 28 and 29.

- Refuel: Clients 1, 2, 3, 4, 5, 7, 8, 9, 11, 13, 14, 19, 23, 25, 26 and 30

- None: Clients 16 and 17

For the case of emergency servicing (Rescue and Recover) all the Client satellites are assumed to be compatible with the Servicers.

\subsection{Servicer Fleet and Simulation Conditions}

Based on the main developments presented in Section 1, parameters are defined for each type of Servicer (Table 6).

\begin{tabular}{|c|c|c|c|c|c|c|c|c|c|}
\hline \multicolumn{10}{|c|}{ Table 6. Servicer parameters } \\
\hline Type & $\begin{array}{c}\text { Total } \\
\text { Mass } \\
{[\mathrm{kg}]}\end{array}$ & $\begin{array}{c}\text { Mass On } \\
\text { Station } \\
{[\mathrm{kg}]}\end{array}$ & $\begin{array}{c}t_{\text {life }} \\
\text { [years] }\end{array}$ & $\begin{array}{c}\text { Cost } \\
{[\text { FY2018 }} \\
\text { M\$] }\end{array}$ & $\begin{array}{l}\Delta V \mathrm{On} \\
\text { Station } \\
{[\mathrm{km} / \mathrm{s}]}\end{array}$ & $\begin{array}{l}\text { Prop. } \\
\text { Orb. } \\
\text { Insert. }\end{array}$ & $\begin{array}{l}\text { Prop. } \\
\text { Station } \\
\text { Keep. }\end{array}$ & $\begin{array}{l}\text { Sellable } \\
\text { Propellant } \\
\text { Mass }[\mathrm{kg}]\end{array}$ & $n_{s}$ \\
\hline Rescue and Recover & 1680.49 & 1057.15 & 20.00 & 172.48 & 1.090 & $\mathrm{BP}$ & $\mathrm{EP}$ & - & 1 \\
\hline Lifetime Extension & 1862.18 & 1171.44 & 20.00 & 179.01 & 3.104 & $\mathrm{BP}$ & $\mathrm{EP}$ & - & 1 \\
\hline Refuel & 5054.78 & 3179.81 & 15.00 & 329.97 & 0.818 & BP & $\mathrm{EP}$ & 1250 (MP) & 1 \\
\hline
\end{tabular}

For Lifetime Extension the Servicer operators must consider the expected mass of the Client being serviced when designing their systems. It is important to note that the $\Delta V$ requirements for station keeping are still the same (around 50 $\mathrm{m} / \mathrm{s}$ per year); however, the total mass of Servicer-Client system will require more propellant mass for the stationkeeping manoeuvres. When such required propellant mass is considered for the Servicer mass alone, a higher $\Delta V$ capacity is presented as it can be noticed in Table 6 , Figure $6 \mathrm{a}(\Delta V)$ and Figure $6 \mathrm{~b}$ (propellant mass).

For planned operations (Lifetime Extension and Refuel) the starting time and orbital slot for the Servicers are based on the first serviced Client. For emergency operations (Rescue and Recover) the Servicers start the operation at the first year of the simulation, in pre-defined orbital slots. The $\Delta V$ estimation for the Servicers follows the same method used for the Clients, considering apogee burn for GTO to GEO transfer. Table 7 presents the main parameters considered for the simulations proposed.

Table 7. Simulation Parameters

\begin{tabular}{lll} 
Parameter & Value & Rationale/Remarks \\
\hline Simulation time & 30 years & - \\
Standard Weibull $\theta$ & 69112.52 years & (Appendix A) \\
Standard Weibull $\beta$ & 0.3607 & (Appendix A) \\
Servicer Weibull $\theta$ & 8338.49 years & Based on Saleh [33] \\
Servicer Weibull $\beta$ & 0.3874 & Based on Saleh [33] \\
Standard TTBE & $40 \%$ of Clients' design life & Estimation based on Graham [39] \\
Standard Discount Rate $(r)$ & $6 \%$ & Estimation based on Graham [39] \\
Standard extension of life & $30 \%$ of $t_{\text {life }}$ for Refuel. & Based on services from Table 1 \\
& $15 \%$ of $t_{\text {life }}$ for Life. Ext. & \\
Life limit for servicing & At $70 \% t_{l i f e}$ for Refuel. & For Refuel and Lifetime Extension \\
& At $50 \% t_{l i f e}$ for Life Ext. & \\
Cost of servicing (servicing charge) & $1.232 \mathrm{M}$ per kg* (Life. Ext.) & Based on the Servicers' cost and \\
& $0.166 \mathrm{M} \$$ per kg* (Refuel.) & sellable capacity/consumable \\
& $3.169 \mathrm{M} \$$ per kg* (Resc. Rec.) & resources** \\
Critical satellite value & $1 \%$ of the total Cost as New & Point at which satellite is \\
& & considered to be of no real value
\end{tabular}


* Price applied to the $\mathrm{kg}$ of resource spent by the Servicer in the entire servicing operation, independent of the application. It considers the propellant spent in the phasing manoeuvre ("delivery"), propellant spent in rendezvous and proximity operations, propellant transferred (if Refuel) and propellant spent in station keeping (if Lifetime Extension).

** The cost of servicing considers the Servicer cost, the available resources (propellant) for the manoeuvres and servicing tasks and the sellable propellant for refuelling. This is a threshold cost estimated to give a break-even condition for the Servicer at a given time (the end of life for the example presented herein).

*** The maximum failure rate used in the stochastic generation of failures is a constraint used by the framework to limit the maximum number of failures allowed to happen in one year of the simulation. However, the value defined does not represent that this number of failures will necessarily happen, as it will be presented in the results.

With exception of Simulation Time, all the simulation parameters presented in Table 7 are considered as individual characteristics of each Client and Servicer satellite. For the purpose of this demonstration though, they are considered the same for the whole Client fleet.

It is important to note that all the parameters from Table 5, Table 6 and Table 7 are expected to be defined by the user/users (Client, Servicer or both) when using the framework in a real case. Since the detailed definition of each parameter would require more extensive information about the user-specific concepts of operation, business plans and heritage knowledge, the values used herein are estimates for the demonstration of the framework. This will be presented in the next section.

As previously mentioned, for the Refuel application the Servicers can be refuelled once they are moved to a refuelling base. Such a base is defined in an orbital slot considered of small commercial interest for geostationary operators. Therefore, the base is considered at the longitude of 170 degrees West (mid-Pacific Ocean). The base is considered static at that position and the Servicers will move to it when it is required. In Figure 4 is also illustrated the location of the Base for refuelling of the Servicers.

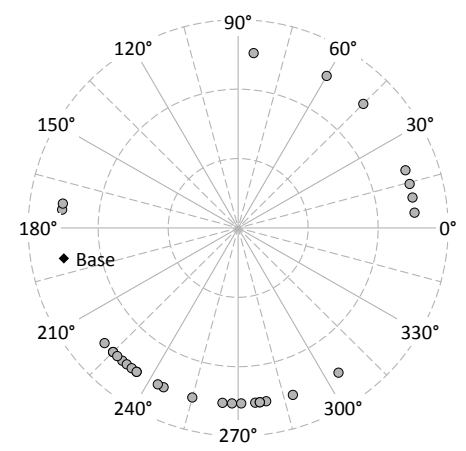

Fig. 4. Longitudinal location of Clients and Base

\section{Results}

As previously discussed, the interaction of Clients and Servicers is not trivial, which reflects the complexity of analysing OOS scenarios. When considering a large fleet this complexity becomes more evident, with multiple metrics to be analysed for multiple satellites. For this reason, an example of each servicing case is isolated from the general results and presented in this section for discussion. In Figure 5 are presented isolated results for the cases of Lifetime Extension and Refuel. In Figure 8 are presented the isolated results for Rescue and Recover. In both figures are presented at least one satellite that was not serviced. In the individual results it is possible to check more clearly the effects of servicing in each of the metrics which are discussed as follows.

\subsection{Lifetime Extension and Refuel}

For the case of planned servicing, the Servicer fleet received 28 requests for servicing of which 22 were effectively serviced. This highlights the characteristic of availability and compatibility of the Servicer fleet.

The effects of Lifetime Extension and Refuel can be noticed respectively by the green and blue curves in Figure 5 . For more clarity in the results, Figure 5 illustrates only the first 18 years of the 30 -year simulation with Client 6 , Client 16 and Client 19 starting their operations at different times through the simulation. In the example, while both Client 6 and Client 19 operate for a longer period than the original design life, there are differences in how the life extension happens.

Looking at the $\Delta V$ curves (Figure 5a), it is possible to verify the condition that maintains the Servicer attached to the Client 6 for the Lifetime Extension case. The Client $\Delta V$ consumption stops for the period of life extension and, after that, the Client continues to use its own fuel towards the end of operation. During the servicing time, the Servicer is in charge of station keeping and orbital corrections, which can be verified in Figure 6a, Servicer 1 (green curve). Still looking at the $\Delta V$, the Client 19 is refuelled around year 11 of the simulation. For this case the $\Delta V$ is recovered due to the refuelling and the Client continues to operate using its own means, allowing the Servicer to be assigned to other servicing operations. The extension in life provided by these two types of servicing will consequently change how the other metrics degrade with time. Figure 5b shows the effects of servicing on the propellant mass of the Client; note that propellant mass is low in the case of Client 6 due to the use of electric propulsion on this satellite. 


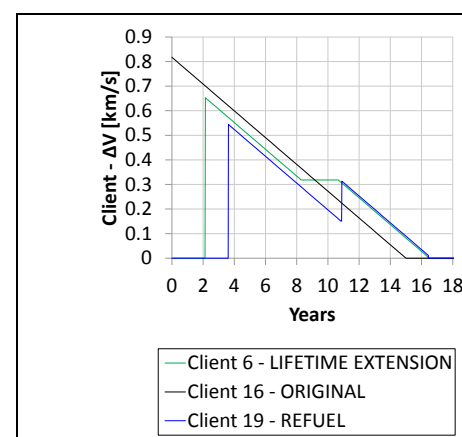

(a)

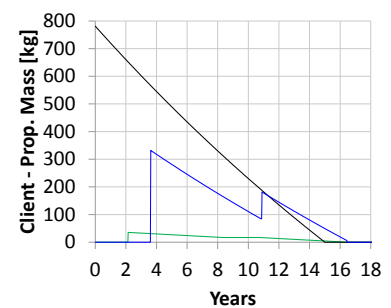

Years

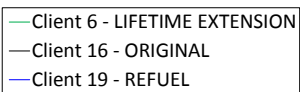

(b)

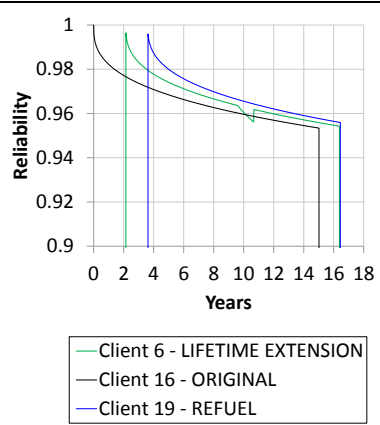

(c)

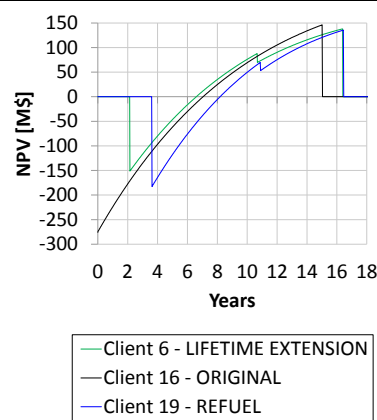

(d)

Fig. 5. Isolated Results - Client metrics - (a) On-Station $\Delta V$, (b) On-Station Propellant Mass, (c) Reliability [R(t)], (d) $N P V$

The Reliability parameters used for this simulation came from a sample of geostationary satellites launched between the years 2000 and 2017 (Appendix 1), using the method presented by Castet and Saleh [33]. Regarding the extended life, even though the systems have longer operational life after servicing, no drastic changes are noticed due to the high reliability of the systems as can be seen in Figure 5c. However, it is also noticeable how Reliability is affected during the periods when the Servicer is attached to the Client. For Client 19, due to the time spent refuelling (between one and two weeks [40]) the Reliability drops for a fairly short time. This effect is more evident for Client 6 . During the extension time, the Servicer is docked to the Client creating a "third system" with different characteristics for mass, dynamics and operation. One trade-off that has been discussed about OOS is related to the possible savings of relaxing systems requirements, which will then affect directly how the reliability curve degrades. Even though the framework allows exploring this trade-off, such analysis would go beyond the scope of the current paper.

Finally, in Figure 5d is presented the Clients' capacity for money generation with time. As described before, it uses the $N P V$ based on stakeholder requirements/characteristics to represent it. Most important, it highlights the effects of servicing as the Client operator pays for it. The main parameters defining such charges from the Servicer perspective are the resources used, in this case fuel and time spent and fuel transferred for refuelling cases. For Client 6, this happens before year 10 when the extension is completed. Client 19 is charged around the same time after the operation is completed. For this metric, in addition to the concept of time value of money, the system degradation with time and how it reduces the capability of revenue generation towards the satellite life is also considered; this is mainly indicated by the curves going "flat" with time. Concurrently with the other metrics presented previously, the framework can be used for trade-offs regarding when to request a service, for how long the life should be extended and which type or servicing should be used.

An example of a satellite that was not serviced is also presented. Such a condition could have occurred due to Client incompatibility or unavailability of the Servicer (which could be either servicing another Client, refuelling itself at the Base, or dead).

Looking from the Servicer perspective (Figure 6), $\Delta V$, propellant masses and $N P V$ are presented for the entire simulation period (30 years). Servicer 1 and Servicer 2 started the operation respectively after the year 5 and year 10 of the simulation, prior to their first servicing operations.

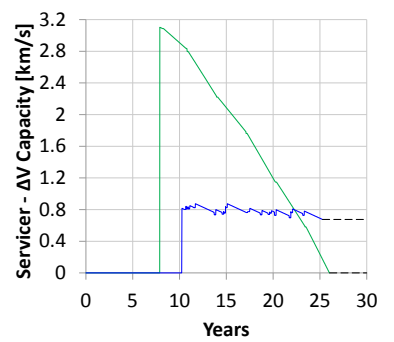

- Servicer 1 - LIFETIME EXTENSION - Servicer 2 - REFUEL

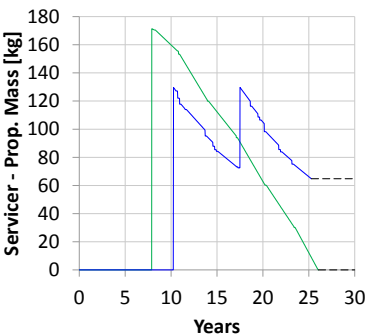

- Servicer 1 - LIFETIME EXTENSION - Servicer 2 - REFUEL

(b)

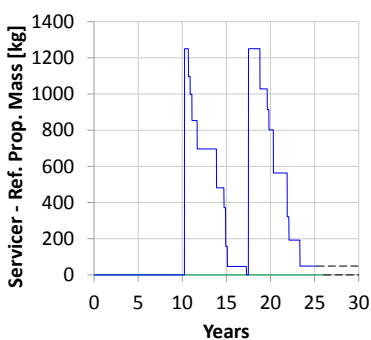

- Servicer 1 - LIFETIME EXTENSION - Servicer 2 - REFUEL

(c)

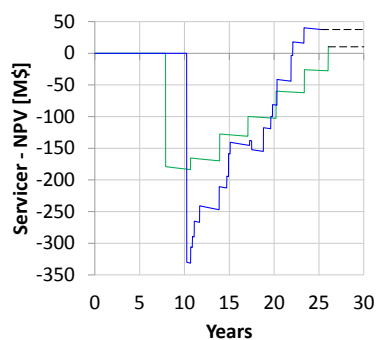

- Servicer 1 - LIFETIME EXTENSION - Servicer 2 - REFUEL (d)

Fig. 6. Servicers metrics - (a) On-Station $\Delta V$, (b) On-Station Propellant Mass, (c) Sellable Propellant Mass - Monopropellant, (d) NPV

From Figure $6 \mathrm{a}$ and Figure $6 \mathrm{~b}$ it can be observed the decrease of $\Delta V$ capacity and the available propellant for manoeuvre and station keeping. Each slope period followed by a small drop in Figure $6 \mathrm{~b}$ represents respectively the periods of station keeping and phasing/servicing manoeuvres. Servicer 2 was considered refillable for both sellable propellant and usable propellant, and the occurrence of such an operation can be observed around year 17. In addition to replenishing its tanks of sellable propellants, it also refuels its propellant for general operations, thus being able to operate through its entire design life (15 years). 
The minor steps-up observed in Figure 6a for Servicer 2 are linked to the Client refuel operations. Since the sellable propellant is considered as a payload and not as usable propellant for the Servicer, each refuel operation reflects in release of mass from the Servicer. However, the available propellant for manoeuvres and station keeping is not consumed for any other task but the phasing manoeuvre as observed in Figure $6 \mathrm{~b}$. This results in a higher $\Delta V$ capacity at each operation and less propellant consumption for manoeuvres and station keeping observed respectively by the stepsup in Figure 6a (Servicer 2) and changes in the curve slope in Figure 6b (Servicer 2).

Servicer 1 has a different concept of operation, moving from one Client to the other and staying for a longer period at each one, in charge of the station keeping manoeuvres. This limits the number of Clients serviced, as presented in Figure 6a and Figure 6b. Additionally, the concept of a "third system" discussed previously changes how the Servicer consumes its propellant mass. Since the propulsion system is in charge of thrusting a much larger mass (Servicer and Client), it consumes propellant more rapidly, as noticed by the changes in the slope for the green curve.

Figure $6 \mathrm{c}$ presents the sellable propellant mass of mono-propellant. From a Servicer perspective, this capacity should be driven by the possible demands of Client refuelling in order to define a proper system and operation concept for refuelling.

Figure 6d presents the Servicers' $N P V$ with time, based only on completed servicing operations. Servicer 1 revenue was around 189M\$ while Servicer 2 recovered at least 367M\$ from its operations. Since the Servicer perspective used in this paper assumed a servicing cost just enough to break-even, the profits from the Servicers were limited. Additionally, the example assumes only one medium size satellite operator which restricts the possible demand for servicing. Other characteristics such as Servicer life and capacity are directly relevant for the exploration of higher profit cases. This highlights the usefulness of this framework relating Servicer and Client, from a Servicer perspective. Fleets from different operators and different sizes can be used as inputs to explore servicing demands and planning.

Finally, in addition to the general metrics results, the framework is able to compare each metric for results with and without servicing. Such comparison considers how each Client performed over its designed life and how the same satellites performed over serviced life (if serviced). This can be used to evaluate how attractive or beneficial the servicing operation is, providing direct outputs for decision making process. As an example, Figure 7 presents the comparison of NPV and Reliability for two serviced Client satellites. The figure illustrates the results the stakeholder would analyse before committing to any decision of servicing or not, one of the main objectives of this framework. The negative value for the comparison of $N P V$ for Client 27 demonstrates that in this case it would not be commercially advisable for the Client operator to proceed with such a servicing strategy.

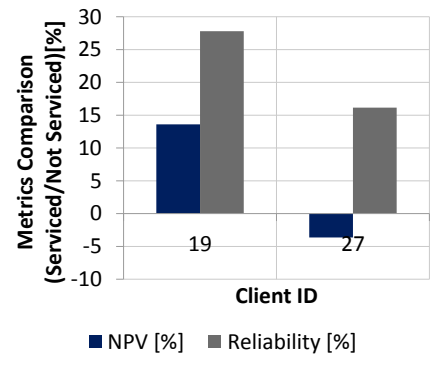

\subsection{Rescue and Recover}

Fig. 7. Servicing Comparison

For the case of emergency servicing, 10 instances of the simulation were run due to the aleatory/stochastic nature of the failure operator. In each instance, different numbers of servicing requests were generated and effectively serviced as presented in Table 8. In the same table are presented for each run the effective rate of failure (number of servicing requests divided by the simulation time) as well as the probability of failure for one of the 30 Client satellites of the fleet (effective rate $_{\text {failure }}$ divided by the number of Client satellites). As described before, the failure rate (rate failure ) presented in Table 7 is used as a constraint of the maximum possible failures in one year of the simulation. The effective rate of failure verifies that the failures generated in a non-deterministic way are below the value defined for the simulation.

Table 8. Rescue and Recover - Servicing request and servicing operations

\begin{tabular}{lllll} 
Run \# & $\begin{array}{l}\text { Servicing } \\
\text { Requests }\end{array}$ & $\begin{array}{l}\text { Servicing } \\
\text { Completed }\end{array}$ & $\begin{array}{l}\text { Effective } \\
\text { rate }_{\text {failure }}\end{array}$ & $\begin{array}{l}\text { Client fail. } \\
\text { probability }\end{array}$ \\
\hline 1 & 9 & 6 & 0.30 & 0.010 \\
2 & 6 & 5 & 0.20 & 0.007 \\
3 & 6 & 4 & 0.20 & 0.007 \\
4 & 13 & 8 & 0.43 & 0.014 \\
5 & 9 & 8 & 0.30 & 0.010 \\
6 & 8 & 8 & 0.27 & 0.009 \\
7 & 10 & 5 & 0.33 & 0.011 \\
8 & 8 & 7 & 0.27 & 0.009 \\
9 & 9 & 5 & 0.30 & 0.010 \\
10 & 10 & 8 & 0.33 & 0.011
\end{tabular}




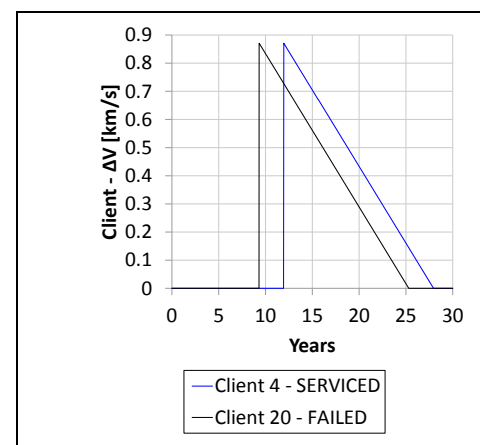

(a)

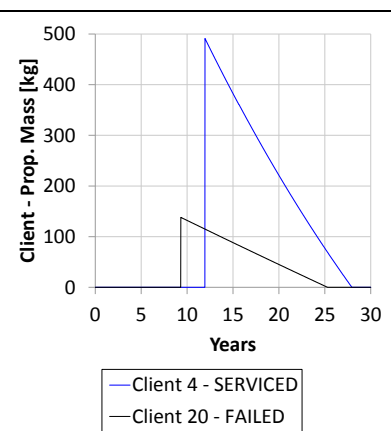

(b)

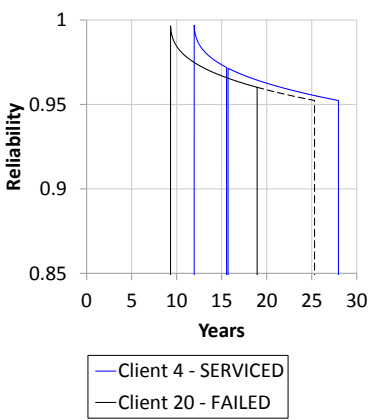

(c)

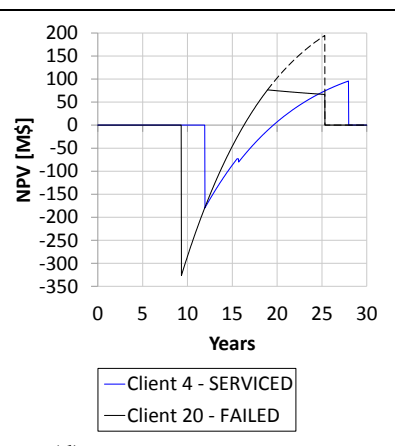

(d)

Fig. 8. Isolated Results (Rescue and Recover run \#10) - Client metrics - (a) On-station $\Delta V$, (b) On-Station Propellant Mass, (c) Reliability [R(t)], (d) NPV

Once more, selected results were isolated from the whole fleet for an example. The same metrics discussed before are presented in Figure 8, showing the results generated from simulation 10. For clarity, the figure presents only the results for a Client that was serviced (Client 4) and a Client that was not serviced (Client 20). Client 4 suffered one failure through the simulation after year 15. For Client 20, after the failure after year 19, a dashed line is used to illustrate how such satellite would continue the operational life if a failure did not occur.

A few assumptions/simplifications are defined for the current example otherwise more specific details would have to be discussed. Yet, the framework is capable of addressing such aspects in a detailed analysis.

First, since the failures are not considered in one particular subsystem for this specific case, it is assumed that, once a Client satellite failed, the operator is not able to commercially use the satellite anymore. For this reason, the failure is assumed to not affect the $\Delta V$ and propellant mass, which explains why no changes are presented in this metric (Figure 8a). On the other hand, the other metrics are affected. Reliability is considered as zero once a Client fails and the operator is not able to use the satellite anymore. Since the satellite is temporarily unusable, no income is generated and $N P V$ decreases due to costs with operations and ground segments (Figure 8d). Considering the $N P V$ decrease, it would be up to the operator to decide whether or not to continue using the satellite, paying for the costs with operation without having an income from that satellite.

This state will continue until the Servicer is assigned and completes the phasing and servicing. The assignment, orbital phasing and servicing take place on a timescale much smaller than the 30 years of the simulation, the reason why this failure interval is not evident in the graphs. An example is presented in Figure 9, illustrating the simulation between years 11 and 18, when Client 4 starts the operation, fails and is serviced between years 15 and 16.

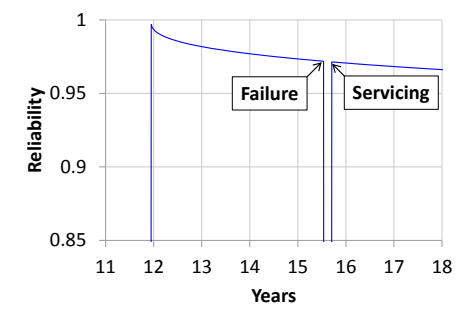

Fig. 9. Rescue and Recover - Reliability - Detail

Another assumption considers that, after servicing, the metric is recovered to the level expected for that point in the lifetime. A further refinement in the failure mode could characterize the failure of a subsystem, reflecting in a partial loss of the metrics affected, instead of zero.

Regarding the servicing charges, for demonstration purposes, only consumables are used to calculate the servicing cost. However, the framework allows the use of additional factors and costs depending on the characteristic of the operation, such as responsiveness and dexterity needed, and the Servicer itself (business model).

Unlike the case of planned servicing, in the Rescue and Recover case it is not possible to set the Servicers to start at one specific time and position when simulating failures in a stochastic/random manner. This reflects in an idle Servicer, spending resources while no servicing requests are generated, as shown in Figure 10. In the figure are presented the results for three different simulations for the visualization of how a Servicer can be requested at any specific time. Each drop in the $\Delta V$ curve (Figure 10a) represents one manoeuvre of the Servicer to respond to a Client failure and can be checked concurrently with Table 8 . 


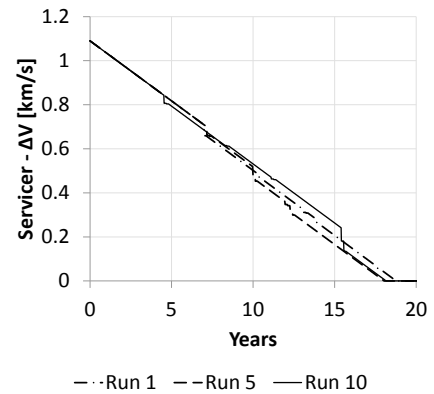

(a)

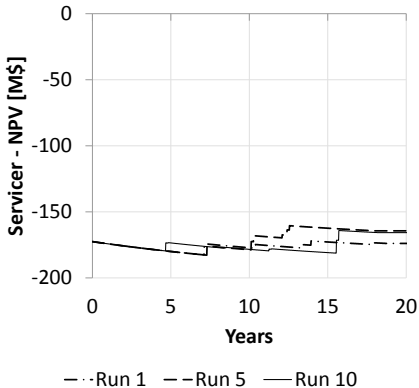

(b)

Fig. 10. Rescue and Recover - Servicer $\triangle V$ and $N P V$

Due to the high reliability and redundancy of space systems nowadays, the case for Rescue and Recover represents a challenge from the Servicer point of view, as it can be observed in Figure 10b. A Servicer aimed for failure remediation should be able to do other tasks in order to be cost efficient. This is already observed in one of the future servicing missions to be launched [8] in which the Servicer is designed not only for failure corrections but also refuelling. As discussed before other factors can be included in the consideration of servicing costs, highlighting another use of this framework.

As previously mentioned, the use of more relaxed Client satellite requirements could be enabled by servicing, especially for the case of Rescue and Recover. This could be simulated in the framework by using different parameters for Reliability and for failure rate. Then this could be a case to be discussed between Servicer and Client operators that would justify servicing satellites exclusively for failure remediation. From the Client operator point of view, a more relaxed design could represent savings in time and cost for the development of satellites. Ellery et al. [41] explore, among other points, the role of high-reliability for different satellite platforms, bringing important guidelines that can be used to simulate more detailed cases of Rescue and Recover.

\section{Discussion}

With the results of this framework it is clear there is a need to consider different metrics of the system to analyse the case of OOS; it is also clear how complex such analysis can be. Different applications of servicing are expected to affect these metrics differently.

One clear point from the results for the hypothetical operator is the fleet scheduling. Even though more than $75 \%$ of the servicing requests were completed in the planned servicing, the number could be increased with a proper arrangement of when the Client satellites are launched. Considering that in a real commercial case Servicers would be in charge of a larger fleet or multiple fleets, this proves to be necessary. The framework could be used for different iterations to find the balance of fleet metrics, launch time and servicing time.

Additionally, how much refuelling or life extension will be achieved with a servicing operation could be better balanced with the NPV of each Client satellite. In this way, any fuel waste or satellite idle time could be avoided.

For the emergency servicing, the consideration of metrics going to zero after a failure is another point to be refined. The consideration of partial failures could more accurately represent real cases of subsystem failures and servicing.

The simulation of more realistic cases, either from Client or Servicer side, encompasses the metrics presented before but also demands the proper characterization of each variable (Table 2) defining the metrics. When one of the primary variables is not provided, the framework uses well-known estimation methods such as cost estimation relationships (CERs) and regression from historic data for satellite parameters. However, considering the limitations and error estimates of such methods, for more representative results the user is expected to provide more refined estimates for such variables.

It should be noted that the context of the simulation will vary, depending on the specific case being considered. Satellite operators have different profiles, demands and concept of operation for their fleets, ranging from technical to commercial/financial aspects. Servicer operators would also be looking at this upcoming market and designing their systems accordingly. Hastings et al. [42] presents a model developed based on behavioural economics which is used to evaluate the long-term effect of servicing in the space industry, bringing results such as trends for servicing cost and trends for servicing adoption. In addition to the current industry/operator needs and interests towards servicing, these results can be used to define variables to explore OOS in the current context and to formulate scenarios to be explored with the framework presented in this paper.

Additionally, different operators might have specific ways of assessing the value of their satellites. Herein, with focus on the upcoming commercial cases of OOS, NPV can be used as main metric for such assessment. However, operators may find value in servicing from the usefulness incorporated to their systems which is not necessarily financially-driven (strategic, scientific or responsive reasons for example). Therefore, the framework also allows the implementation of metrics defined by the user to help it in the tailoring of the simulation for each profile. Such additional metrics can be used in parallel with the metrics presented in this work, not affecting the functioning of the framework. As standard examples found in the literature, Utility presented by Saleh et al. [38] can be used in parallel with the current metrics depending on the user's concept of operation and requirements. 
Due to length limitation, the example presented here considers only the case of Lifetime Extension, Refuel and Rescue and Recover but the methodology can also consider cases such as Maintenance and Repair (Payload Augmentation) and even Re-orbit/De-orbit (Active Debris Removal).

\subsection{Satellite Design and Operation}

In this section are discussed some immediate explorations that are possible using the results from the framework presented in the previous sections.

- Extension vs. Refuel: Even though Lifetime Extension and Refuel have the same final result, the implications in selecting one of them are different. For the former, no major changes in the design of the Client satellite are required as this application relies more on the Servicer side (grappling, berthing and docking mechanisms) [2,3]. Mechanical interfaces already present on the Client satellite such as the interface with the launcher are suitable for this application. On the other hand, the Refuel is likely to demand changes in the propulsive systems such as fluidic interfaces, and eventual limitations to the types of fuel. Some of these subsystems were already demonstrated and have a maturity around TRL 7 and 8 [43-45]. Despite requiring changes in the Client design, the Refuel application allows the Servicer to be more flexible in its operation (multiple Clients) which could reflect in a less costly servicing. The application of Lifetime Extension, however, primarily requires the Servicer to be dedicated to one Client at a time, which could lead to a more costly servicing. Another point to be noted regards the chance of the Client operation being affected by the presence of the Servicer, as demonstrated in the example. All the presented metrics are used to explore a suitable option to an operator.

- Mass allocation: The specific mass allocation of the payload and the propulsion subsystem is another design point to take advantage of OOS. The current mass allocated before the satellite commissioning for the propulsion subsystem and fuel is between 50\% and 70\% of the total launch mass for geostationary satellites. Reducing this mass would allow the operator to add more payload (and auxiliary subsystems such as power) to the satellite, increasing potential revenue. The reduced lifetime could then be balanced by either Lifetime Extension or Refuel as discussed before. All the metrics discussed in the paper are the main guidelines to perform such decision-making, in addition to metrics from the operator. This also requires a concurrent evaluation with the Servicer side to guarantee the suitable operation of the Client satellites.

- Flexible payloads: Flexible payloads have been designed to be adaptable to different conditions such as changeable coverage requirements, and target user markets, and are capable of changes during the satellite life. Even though such payloads are able to keep generating Income and NPV at (assumed) better conditions than a conventional payload, the satellite is still dependent on the propulsion subsystem to make the most of such a payload. The selection of one of the types of servicing discussed before is an option to reach this, considering the two previous points discussed. For this case, the $N P V$ metric has a major role to evaluate this design option.

- Expandable and hosted payloads: Either for the case of a Client operating for its initial design life or for an extended life, the capacity of operating under different conditions such as market changes is one area that can also be explored in the design of a serviceable Client. The addition of extra payloads on demand is one direct option. In this case the electrical-mechanical interface design would have to be revised for a "plug-and-play" payload. Furthermore, the possibility of hosting third party payloads (from smaller operators for example) might allow the operator to extract more income from a satellite, independently from the main mission of the system. For this case, the capabilities of Servicers from Rescue and Recover are the main point to explore in the relation of Servicer and Client. Once the new payload is added, the system is expected to change its value and usefulness from the operator point of view so, in addition to $N P V$, specific user defined metrics can be useful in the evaluation of this application. The concept of hosted payload is already used by some satellite operators [46] with plans to use OOS as an enabler for payload incorporation after launch [47].

- Multi-application: The use of a Servicer for multiple servicing applications is another exploration subject of interest. When considering cases with low demand or unpredictable demand, a Servicer could alternate between its tasks, going, for example, from a Client rescue to another Client refuelling or from a Client life extension to the augmentation of the payload of another Client. For this case, observing the periods when the Servicer is idle is relevant to explore the possibility of performing extra tasks. The use of a Servicer with multiple applications is already anticipated by the upcoming operators such as SpaceLogistics Services [48] and Space Infrastructure Services [40].

\section{Conclusions}

A framework using Agent-Based Modelling Simulation was proposed to relate Client and Servicer sides for different applications of On-Orbit Servicing. A hypothetical satellite operator was used as an example to explore applications of Lifetime Extension, Refuel, and Rescue and Recover. Conditions extracted from real satellite operators and Servicers based on the spacecraft currently in development were used for the demonstration of the framework.

Analysis of OOS with focus on only one side, Client or Servicer, might lead to misguided representation of OOS. Furthermore, considering only individual aspects of a satellite operation, such as deltaV-only, reliability-only or financial-only can also leave out a portion of the analysis parameters for OOS. The integrated relation of Client and Servicer and the concurrent evaluation of different metrics of the systems, attributes included in the framework, helps to build a more solid analysis of OOS applications in early stages of the design. 


\section{Acknowledgements}

The researcher is sponsored by the Brazilian Ministry of Science and Technology and Innovation through the process 202687-2014/7 (CsF-AEB).

\section{Appendix A}

\section{Reliability: Weibull Function Parameters}

- Sample start date: 01/01/2000

- Sample censor date: 31/12/2017

- Number of satellites: 535

- $\beta: 0.3607$

- $\theta$ : 69112.52 years

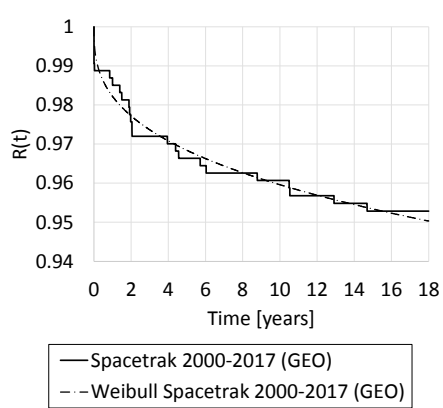

Fig. A1. Weibull distribution and fit

\section{References}

[1] T. Matos de Carvalho, J. Kingston, On-Orbit Servicing Readiness Assessment: The Servicer Perspective, in: Proc. 67th Int. Astronaut. Congr., 2016: p. 10.

[2] A. Flores-Abad, O. Ma, K. Pham, S. Ulrich, A review of space robotics technologies for on-orbit servicing, Prog. Aerosp. Sci. 68 (2014) 1-26. doi:10.1016/j.paerosci.2014.03.002.

[3] G.G. Gefke, B. Reed, Advances in Robotic Servicing Technology Development, Am. Inst. Aeronaut. Astronaut. (2015) 1-9.

[4] A. Pasztor, WSJ - Orbital ATK and Intelsat Set to Sign Satellite Servicing Pact, Wall Str. J. (2016). http://www.wsj.com/articles/orbital-atk-and-intelsat-set-to-sign-satellite-servicing-pact-1457444904.

[5] Orbital ATK, Orbital ATK - Press Release - Orbital ATK Receives Order for Second In-Orbit Satellite Servicing Vehicle, (2018). https://www.orbitalatk.com/news-room/release.asp?prid=317 (accessed February 21, 2018).

[6] J. Foust, SpaceNews.com - Effective Space signs first contract for satellite life extension services, Spacenews.Com. (2018). http://spacenews.com/effective-space-signs-first-contract-for-satellite-life-extensionservices/ (accessed February 21, 2018).

[7] B.B. Reed, R.C. Smith, B. Naasz, J. Pellegrino, C. Bacon, The Restore-L servicing mission, in: AIAA Sp. Astronaut. Forum Expo. Sp. 2016, 2016.

[8] DARPA, Program Solicitation - DARPA-PS-16-01 for Robotic Servicing of Geosynchronous Satellites (RSGS), Arlington, 2016.

[9] C. Henry, SpaceNews.com - Airbus to challenge SSL, Orbital ATK with new space tug business, Spacenews.Com. (2017). http://spacenews.com/airbus-to-challenge-ssl-orbital-atk-with-new-space-tugbusiness/ (accessed February 13, 2018).

[10] H. Sommer, Novel space infrastructure concepts from LEO to GEO, in: AIAA 57th Int. Astronaut. Congr. IAC 2006, 2006.

[11] Y.N. Razoumny, D.B. Spencer, B. Agrawal, J. Kreisel, T. Yasaka, S.A. Koupreev, V. Razoumny, Y. Makarov, The concept of On-Orbit-Servicing for next generation space system development and its key technologies, in: 68th Int. Astronaut. Congr., International Astronautical Federation, 2017.

[12] J. Kreisel, On-Orbit Servicing of Satellites (OOS): Its Potential Market \& Impact, in: 7th ESA Work. Adv. Sp. Technol. Robot. Autom. "ASTRA 2002," 2002: pp. 1-9.

[13] M. Kortmann, S. Rühl, J. Weise, J. Kreisel, T.A. Schervan, H. Schmidt, A. Dafnis, Building block-based "iBOSS" approach: Fully modular systems with standard interface to enhance future satellites, in: 66th Int. Astronaut. Congr. 2015, International Astronautical Federation, IAF, 2015: pp. 9261-9271.

[14] DLR, iBOSS - intelligent Building Blocks for On-Orbit Satellite Servicing and Assembly, (2018). http://www.iboss-satellites.com.

[15] Orbital ATK - Fact Sheet - Space Logistics Services, (2017).

[16] Orbital ATK - Fact Sheet - Mission Extension Vehicle (MEV), (2017).

[17] SSL/MDA - Press Release - MDA announces On-Orbit Satellite Servicing business formation and contract awards for spacecraft and first life extension customer, (2017). 
https://www.sslmda.com/html/pressreleases/pr20170628.html (accessed February 21, 2018).

[18] G. Hirzinger, K. Landzettel, B. Brunner, M. Fischer, C. Preusche, D. Reintsema, A. Albu-Schäffer, G. Schreiber, B.-M. Steinmetz, DLR's robotics technologies for on-orbit servicing, Adv. Robot. 18 (2004) 139174. doi:10.1163/156855304322758006.

[19] S. Cresto Aleina, N. Viola, F. Stesina, M.A. Viscio, S. Ferraris, Reusable space tug concept and mission, Acta Astronaut. 128 (2016) 21-32. doi:10.1016/j.actaastro.2016.07.003.

[20] A. Ellery, An introduction to space robotics, 1st ed., Springer-Verlag New York, London, 2000.

[21] B.R. Sullivan, D.L. Akin, A survey of serviceable spacecraft failures, in: AIAA Sp. 2001 Conf. Expo., 2001: pp. 1-8. doi:10.2514/6.2001-4540.

[22] C. Reynerson, Spacecraft Modular Architecture Design for on-orbit servicing, in: Sp. Technol. Conf. Expo., American Institute of Aeronautics and Astronautics, Reston, Virigina, 1999. doi:10.1109/AERO.2000.878426.

[23] P.O. Siebers, C.M. Macal, J. Garnett, D. Buxton, M. Pidd, Discrete-event simulation is dead, long live agentbased simulation!, J. Simul. 4 (2010) 204-210. doi:10.1057/jos.2010.14.

[24] C.M. Macal, M.J. North, Tutorial on agent-based modelling and simulation, J. Simul. 4 (2010) 151-162. doi:10.1057/jos.2010.3.

[25] C.M. Macal, M.J. North, Agent-based modeling and simulation: ABMS examples, in: 2008 Winter Simul. Conf., IEEE, 2008: pp. 101-112. doi:10.1109/WSC.2008.4736060.

[26] R. Janz, F. Cheng, J. Borrego, COSEMS - A study simulation of space logistics, 2nd Sp. Logist. Symp. (1988). doi:doi:10.2514/6.1988-4710.

[27] M.G. Richards, N.B. Shah, D.E. Hastings, Agent Model of On-Orbit Servicing Based on Orbital Transfers, Proc. AIAA Sp. Conf. (2007) 1-12. doi:10.2514/6.2007-6115.

[28] E.S. Lamassoure, A Framework to Account for Flexibility in Modeling the Value of On-Orbit Servicing for Space Systems, Massachusetts Institute of Technology, 2001.

[29] J.H. Saleh, E.S. Lamassoure, D.E. Hastings, D.J. Newman, Flexibility and the Value of On-Orbit Servicing: New Customer-Centric Perspective, J. Spacecr. Rockets. 40 (2003) 279-291. doi:10.2514/2.3944.

[30] Seradata, SpaceTrak, Seradata. (2018). http://www.seradata.com/.

[31] R. Bate, Fundamentals of astrodynamics, Dover Publications, New York, 1971.

[32] D.A. Vallado, W.D. McClain, Fundamentals of astrodynamics and applications, Springer, 2007.

[33] J.-F. Castet, J.H. Saleh, Satellite Reliability: Statistical Data Analysis and Modeling, J. Spacecr. Rockets. 46 (2009) 1065-1076. doi:10.2514/1.42243.

[34] J.R. Wertz, D.F. Everett, J.J. Puschell, Space Mission Engineering: The New Smad, Microcosm Astronautics, Hawthorne, 2011.

[35] J.W. Dankanich, G.R. Woodcock, Electric Propulsion Performance from Geo-transfer to Geosynchronous Orbits, 30th Int. Electr. Propuls. Conf. (2007) 287.

[36] D. Losa, High vs Low Thrust Station Keeping Maneuver Planning for Geostationary Satellite, Ecole Nationale Superieure des Mines de Paris, 2007.

[37] F. Topputo, F. Bernelli-Zazzera, Optimal Low-Thrust Station Keeping of Geostationary Satellites, in: 3rd CEAS Air\&sp. Conf. 21st AIDAA Congr., 2011.

[38] J.H. Saleh, D.E. Hastings, D.J. Newman, Weaving time into system architecture: satellite cost per operational day and optimal design lifetime, Acta Astronaut. 54 (2004) 413-431. doi:10.1016/S0094-5765(03)00161-9.

[39] A.R. Graham, J. Kingston, Assessment of the commercial viability of selected options for on-orbit servicing (OOS), Acta Astronaut. 117 (2015) 38-48. doi:10.1016/j.actaastro.2015.07.023.

[40] SIS, Space Infrastructure Services (SIS), (2018). http://spaceinfrastructureservices.com/ (accessed February 12, 2018).

[41] A. Ellery, J. Kreisel, B. Sommer, The case for robotic on-orbit servicing of spacecraft: Spacecraft reliability is a myth, Acta Astronaut. 63 (2008) 632-648. doi:10.1016/j.actaastro.2008.01.042.

[42] D.E. Hastings, B.L. Putbrese, P.A. La Tour, When will on-orbit servicing be part of the space enterprise?, Acta Astronaut. 127 (2016) 655-666. doi:10.1016/j.actaastro.2016.07.007.

[43] S. Rotenberger, D. SooHoo, G. Abraham, Orbital Express fluid transfer demonstration system, in: R.T. Howard, P. Motaghedi (Eds.), SPIE Def. Secur. Symp., International Society for Optics and Photonics, 2008: pp. 695808-695808-9. doi:10.1117/12.783948.

[44] L. Poynter, R. Rembala, P.A. Keenan, A. Ogilvie, The advancement of robotic servicing capabilities through Dextre utilization and technology demonstration on the International Space Station, in: International Astronautical Federation, IAF, 2013: pp. 3700-3708.

[45] NASA, Robotic Refueling Mission, https://www.nasa.gov/mission_pages/station/research/experiments/778.html (accessed August 15, 2017).

[46] SES, Hosted Payloads, SES Gov. Solut. (2018). https://ses-gs.com/solutions/fixed-sat-solutions/hostedpayloads/ (accessed February 16, 2018).

[47] SES ready to invest in reusable rockets, in-orbit satellite servicing - SpaceNews.com, (2016). http://spacenews.com/ses-ready-to-invest-in-reusable-rockets-in-orbit-satellite-servicing/ (accessed February 16, 2018).

[48] SpaceLogistics, Fact Sheet - SpaceLogistics Services, (2018). 
2018-10-26

\section{Establishing a framework to explore the Servicer-Client relationship in On-Orbit Servicing}

Matos de Carvalho, Tiago Henrique

Elsevier

Matos de Carvalho TH, Kingston J. (2018) Establishing a framework to explore the Servicer-Client relationship in On-Orbit Servicing. Acta Astronautica, Volume 153, December 2018, pp. 109-121

https://doi.org/10.1016/j.actaastro.2018.10.040

Downloaded from Cranfield Library Services E-Repository 